\title{
A case study of glycogen storage disease type la presenting with multiple hepatocellular adenomas: an analysis by gadolinium ethoxybenzyl-diethylenetriamine-pentaacetic acid magnetic resonance imaging
}

\author{
Xiaoming Li ${ }^{1 \#}$, Hui Jing ${ }^{2 \#}$, Lin Cheng ${ }^{1}, \mathrm{Jie} \mathrm{Xia}^{3}$, Jian Wang ${ }^{1}, \mathrm{Qing}^{4}{ }^{4}$, Chen Liu ${ }^{1}$, Ping Cai $^{1}$ \\ ${ }^{1}$ Department of Radiology, Southwest Hospital, Third Military Medical University (Army Medical University), Chongqing, China; ${ }^{2}$ Department of \\ Radiology, Shan Xi Medical University, Taiyuan, China; ${ }^{3}$ Department of Infectious Diseases, Southwest Hospital, Third Military Medical University \\ (Army Medical University), Chongqing, China; ${ }^{4}$ Magnetic Resonance Collaborations, Siemens Healthcare Ltd., Shanghai, China
}

"These authors contributed equally to this work.

Correspondence to: Chen Liu; Ping Cai. Department of Radiology, Southwest Hospital, Third Military Medical University (Army Medical University), Chongqing 400038, China. Email: liuchen@aifmri.com; 530305942@qq.com.

\begin{abstract}
Glycogen storage disease type Ia (GSD Ia) is a rare disease caused by a deficiency of hepatic glucose-6-phosphatase (G6Pase). Here, we report a 17-year-old Chinese boy with GSD Ia. Clinical manifestations of the patient included hepatomegaly, growth retardation, doll face, and biochemical abnormalities, including hypoglycaemia, hyperuricaemia, and hyperlipidaemia. The computed tomography (CT) and gadolinium ethoxybenzyl-diethylenetriamine-pentaacetic acid (Gd-EOB-DTPA) magnetic resonance imaging (MRI) revealed multiple masses in the left and right hemiliver. These masses presented as different dynamic enhanced patterns in the Gd-EOB-DTPA MRI. In addition, a large amount of glycogen deposit was detected in the liver tissue biopsy. Liver puncture confirmed that the masses were hepatocellular adenomas (HCAs). Genetic analyses confirmed the presence of liver metabolic disease, and the final clinical diagnostic was GSD Ia. The patient's clinical manifestations were significantly improved following regular treatment with raw corn starch for 9 months. Unfortunately, it was suspected that parts of the adenoma had undergone malignant transformation.
\end{abstract}

Keywords: Glycogen storage disease (GSD); gadolinium ethoxybenzyl-diethylenetriamine-pentaacetic acid (GdEOB-DTPA); magnetic resonance imaging (MRI); adenoma; gene

Submitted Jun 09, 2020. Accepted for publication Nov 06, 2020.

doi: 10.21037/qims-20-746

View this article at: http://dx.doi.org/10.21037/qims-20-746

\section{Introduction}

Glycogen storage disease type Ia (GSD Ia) is an extremely rare autosomal recessive inherited disorder affecting glycometabolism, with a prevalence of 1 in 100,000 (1). Deficiency of the enzyme glucose 6-phophatase (G6Pase) leads to abnormal glycogen metabolism, which then causes abnormal deposits of glycogen in the endoplasmic reticulum cavity. European research guidelines recommend that the diagnosis of GSD Ia should be based on clinical manifestations, imaging, and abnormal biochemical indicators (2). Diet and adjuvant drug therapy are the main forms of treatment for GSD type I. The objective is to maintain blood glucose levels and prevent long-term complications, including the development of hepatocellular adenomas (HCAs) and hepatocellular carcinomas (HCCs) (3). Although the use of magnetic resonance imaging (MRI) in 

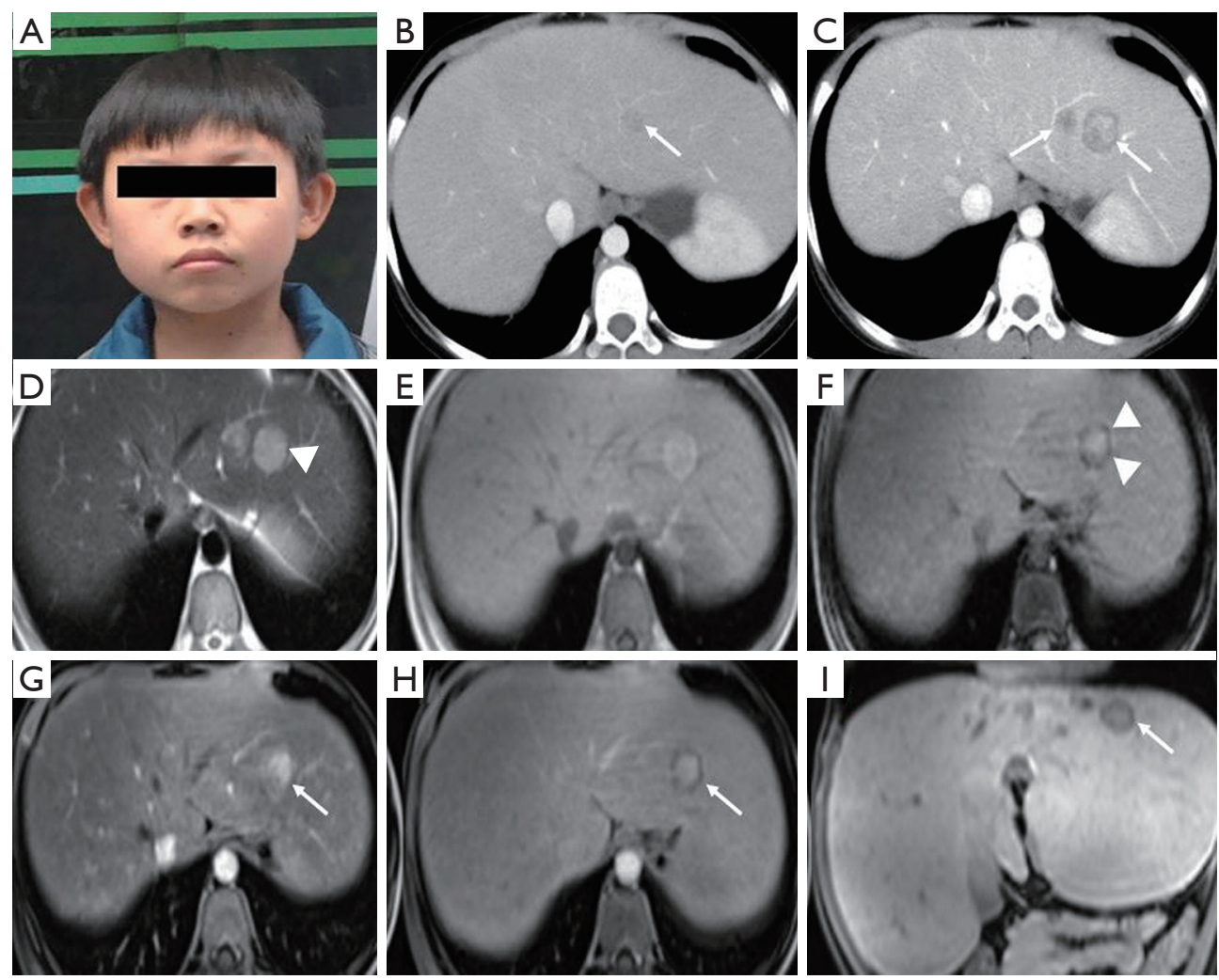

Figure 1 The computed tomography (CT) and magnetic resonance imagining (MRI) images of a 17-year-old male patient with Glycogen storage disease type Ia and hepatocellular adenomas. (A) A photograph of the patient presenting with doll face, growth retardation, and absence of male sexual development. (B,C,D,E,F) The contrast-enhanced CT results performed at time intervals of 15 months. Examination on July 1, 2015 showed a low-density nodule in segment II (white arrow, B). At the patient's second examination, the number and diameter of the nodules had increased (white arrows, C). The lesion (no. 2, white triangle, D) was hyperintense on T2WI. The lesion was hyperintense on in-phase analysis (E). Perilesional signal (white triangles) was decreased on opposed-phase analysis (F). (G,H,I) The dynamic enhanced MRI. The lesion was significantly enhanced in the arterial phase (white arrow, G), and persisted to the delayed phase (white arrow, H) in comparison with adjacent hepatocytes. (I) Coronary hepatobiliary phase (HBP; white arrow) showed enlarged liver volume, and the nodule was hypointense but mixed with a slightly hyperintense interior.

the detection of HCAs has been reported in the literature (4-6), to our knowledge, this is the first case to include a comprehensive dataset, including clinical signs, laboratory tests, imaging manifestations, pathology, gene sequencing, and follow-up results.

\section{Case presentation}

A 17-year-old boy presented with growth retardation and was initially admitted for short stature. Physical examination revealed weight loss, lack of male sexual development, facial signs of chronic liver disease (Figure 1A), and an enlarged liver. Fasting blood glucose levels $(3.57 \mathrm{mmol} / \mathrm{L})$ were slightly decreased. Platelet count $\left(374 \times 10^{9} \mathrm{~g} / \mathrm{L}\right)$, alkaline phosphatase (274 IU/L), glutamyl transpeptidase (233 IU/L), glutamic oxaloacetic transaminase (115 IU/L), alanine transaminase $(71 \mathrm{IU} / \mathrm{L})$, triglycerides $(5.76 \mathrm{mmol} / \mathrm{L})$, total cholesterol $(6.54 \mathrm{mmol} / \mathrm{L})$, lactic acid $(9.9 \mathrm{mmol} / \mathrm{L})$, albumin $(51.20 \mathrm{~g} / \mathrm{L})$, anion gap $(24 \mathrm{mmol} / \mathrm{L})$, and uric acid (477 $\mu \mathrm{mol} / \mathrm{L}$ ) levels were all elevated. He tested positive for ketone bodies. Other indicators, such as alpha fetoprotein and abnormal prothrombin, were normal. He also tested positive for hepatitis B virus (HBV) markers and had been undergoing antiretroviral therapy for the past 16 years.

The contrast-enhanced abdominal computed tomography (CT) examination at the time of admission (Figure 1B) showed multiple low-density nodules, and the largest nodule was located in segment II of the liver. Abdominal CT scans 
Table 1 The gadolinium ethoxybenzyl-diethylenetriamine-pentaacetic acid magnetic resonance imaging (Gd-EOB-DTPA MRI) findings of multiple adenomas

\begin{tabular}{|c|c|c|c|c|c|c|c|c|}
\hline No. & Location & Size $(\mathrm{cm})$ & T1WI & $\mathrm{T} 2 \mathrm{WI}$ & Arterial-phase & Delayed-phase & Hepatobiliary phase & $\begin{array}{l}\text { Size after } 9 \text { months }(\mathrm{cm}) \\
\text { and hepatobiliary phase }\end{array}$ \\
\hline 1 & II & 1.8 & High & High & High & High & $\begin{array}{l}\text { Low signal, } \\
\text { mixed with high signal }\end{array}$ & Unchanged \\
\hline 2 & II & 2.3 & High & High & High & High & $\begin{array}{l}\text { Low signal, } \\
\text { mixed with high signal }\end{array}$ & 2.7 \\
\hline 4 & III & 1.1 & High & High & High & High & Iso & 2.1 , hypointensity \\
\hline 5 & III & 0.6 & Equal & Equal & High & Low & Iso & 1.0 , hypointensity \\
\hline 6 & III & 0.7 & Low & Equal & High & High & Low & Unchanged \\
\hline 7 & IV & 0.8 & Low & High & High & Low & Low & Unchanged \\
\hline 10 & IV & 1.2 & Low & High & High & High & Low & Unchanged \\
\hline 11 & VII & 0.7 & Low & High & High & Low & Low & Unchanged \\
\hline 12 & VII & 0.5 & High & High & High & Low & Low & Unchanged \\
\hline 13 & VIII & 1.1 & High & Equal & High & High & $\begin{array}{l}\text { Low signal, } \\
\text { mixed with high signal }\end{array}$ & Unchanged \\
\hline
\end{tabular}

performed 15 months later (Figure $1 C$ ) revealed that the number and diameter of the nodules had increased.

The gadolinium ethoxybenzyl-diethylenetriaminepentaacetic acid (Gd-EOB-DTPA) MRI (Figure 1D,E,F,G,H,I) revealed an enlarged liver with fat infiltration. Thirteen abnormal rounded nodules were found in the left and right hemiliver with different diameters and signal features. Details of the Gd-EOB-DTPA MRI findings are shown in Table 1. These hypervascular features are commonly observed in different histological types of liver tumors (7). Therefore, to establish an exact diagnosis of GSD, systematic clinical characteristics including the enlarged liver, developmental retardation, and laboratory examinations were used together with the same hypervascular MRI imaging presentations.

Liver puncture was performed vertically into the liver (about $3 \mathrm{~cm}$ deep), and soft liver tissues $(1 \mathrm{~cm}$ ) were rapidly extracted. Staining of the liver tissues with periodic acidSchiff (PAS) showed large amounts of glycogen deposition in the hepatocytes (Figure 2A). The pathology of one of the nodules was confirmed to be HCA (Figure 2B). Analyses of the genes (Figure 2C) related to liver metabolic diseases suggested that there was a homozygous mutation of the glucose 6-phophatase catalytic (G6PC) gene on exon 5 (rs80356484), c.G648 T. This caused CTG to change to CTT (p. Leu216L) and created a new splicing site located 91 base pairs (bp) downstream of the authentic splice site. The patient's parents were found to be heterozygous for c.G648 T. Therefore, the final clinical diagnosis for this patient was GSD Ia.

The patient was prescribed $50-100 \mathrm{~g}$ of raw corn starch four times a day (every 4-6 hours). After 9 months, the patient had grown by $10 \mathrm{~cm}$ in height, and secondary sexual characteristics had begun to develop, including facial hair, pubic hair, and seminal emission. However, Gd-EOBDTPA MRI revealed that the diameter of the four lesions in segments II and III were larger than previously observed. In addition, two of the lesions were now hypointense, while in the previous hepatobiliary phase (HBP), they were hyperintense (Figure 3).

\section{Discussion}

GSD Ia is a rare inherited metabolic disorder. Due to the lack of G6Pases, glucose 6-phosphates cannot be further hydrolysed into glucose, and thus excessive amounts of glycogen are accumulated. This leads to hepatomegaly (8). 

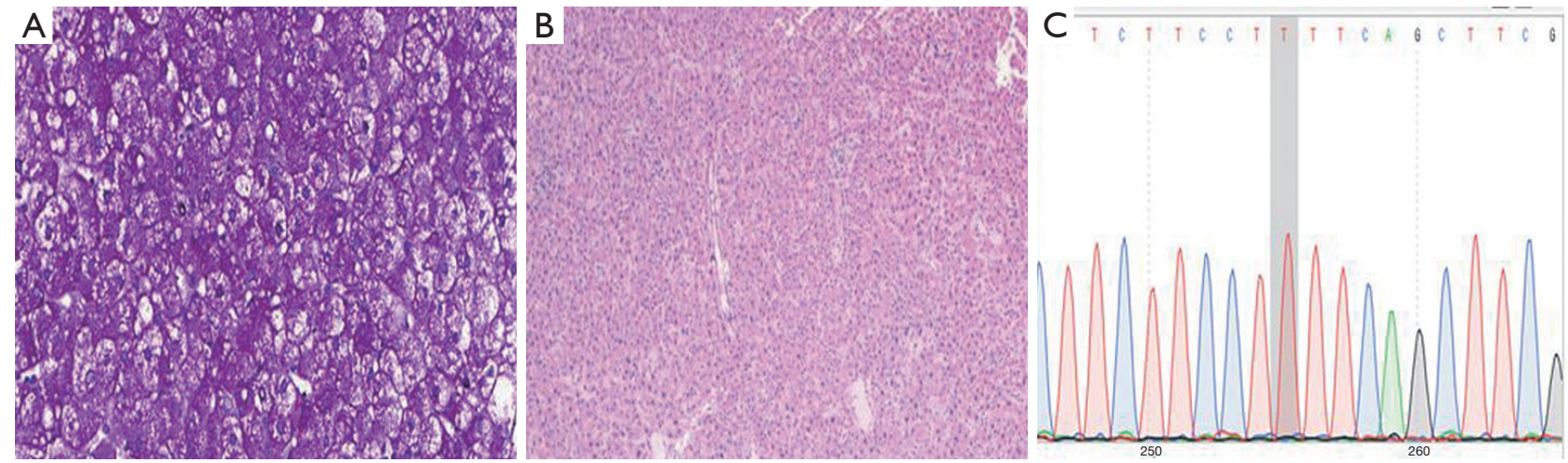

Figure 2 Pathological examinations. (A) Periodic acid-Schiff (PAS) staining of the specimen showed a large amount of glycogen deposition in the hepatocytes (400x magnification). (B) Hematoxylin and eosin (HE) staining of the biopsy showed the presence of tumor cells (400x magnification). (C) The pathogenic mutation caused CTG to change to CTT in exon V at the 17th chromosome.
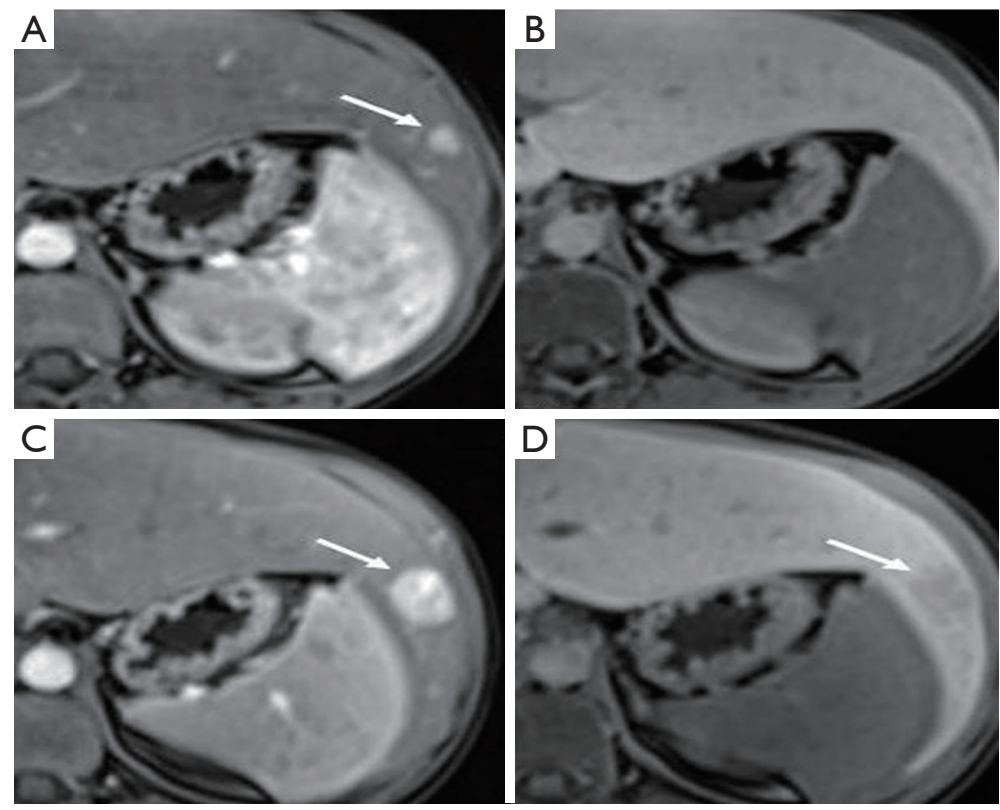

Figure 3 The gadolinium ethoxybenzyl-diethylenetriamine-pentaacetic acid magnetic resonance imaging (Gd-EOB-DTPA MRI) scans at the time of admission and at 9-month follow-up. (A,B) Scans taken on October 30th, 2016 at the time of admission and (C,D) scans taken on July 29, 2017 after 9 months of medical treatment. The lesion (white arrow, referred to as no. 4 adenoma in Table 1) presented with obvious enhancement in the arterial phase (A), and showed isointensity on hepatobiliary phase (HBP) in comparison with adjacent hepatocytes (B). After 9 months' treatment, the diameter of the lesion (C) had grown larger in size compared to (A) and showed hypointensity on HBP (D).

Whole gene sequencing of the G6PC gene can be used to confirm the diagnosis of GSD Ia (9), which accounts for about $80 \%$ of GSD type I. Genetic mutations at certain sites of G6PC are related to different ethnic groups. For example, the mutation c.247C > T (p. Arg83Cys) has a high incidence in the Caucasian (32\%) and Jewish (96\%) communities, whereas the mutation of c.378_379dupTA (p. Tyr128Thrfs*3) is most common in the Hispanic population $(50 \%)$. The genetic variation in our case study is a homozygous point mutation on exon $\mathrm{V}$ at the 17 th chromosome $(648 \mathrm{G}>\mathrm{T}$, p. Leu216L), and has a high prevalence in the Chinese (36-40\%) and Japanese (85-88\%) 
populations (3).

Based on the molecular types of HCA, it can be classified into four subgroups with different imaging features, including hepatocyte nuclear factor 1A HCA (HHCA), inflammatory HCA (IHCA), activating $\beta$-catenin HCA ( $\beta$-catenin HCA), and unclassified HCA (UHCA) (10). Approximately $16-75 \%$ of HCAs are associated with GSD type I (3). The signal intensity of HCAs varies on T1weighted imaging (T1WI) and T2-weighted imaging (T2WI) MRI sequences, which may be explained by variations in adipose, hemorrhage, or glycogen deposition. Decreased signal intensity on T1WI opposed-phase images can be caused by intratumoral fat deposition presented in HHCAs. The "atoll sign" (peripheral high signal intensity) on T2WI has been proposed to be a characteristic finding of IHCAs (11). The obscure scar may be a possible sign of the BHCAs, but the specificity of these features remains very low (12). All types of HCAs show hypervascularization in the arterial phase. Different dynamic enhanced patterns, including wash-in, wash-out, and lasting enhancements, may be caused by different types of pathology. Generally, IHCAs and $\beta$-catenin HCAs show lasting enhancement, while HHCAs and UHCAs show wash-in and wash-out patterns (11). HCAs typically appear hypointense on HBP which is also correlated with a low level of organic anion transporting polypeptide (OATP) expression. Yet, several studies have reported HCAs showing iso- or hyperintensity on HBP, especially in IHCAs and BHCAs (13). This may be related to the uptake of the contrast agent due to the overexpression of OATP. Secondly, it may be explained by the combination of tumor hyperintensity on precontrast T1WI and underlying steatosis (12).

It is worth nothing that this disease should be differentiated from focal nodular hyperplasia (FNH), a highly differentiated HCC. While HCAs are easily confused with FNH, the treatments for HCAs and FNH are different. The majority of FNH cases only require follow-up observation, whereas HCAs require clinical intervention. Approximately $90 \%$ of $\mathrm{FNH}$ and some HCA cases show hyperintensity in the HBP. The signal of FNH on T2WI imaging is close to that of normal liver parenchyma and often associated with a central scar (14). Intratumoral fat deposition and the atoll sign are typical features in HCAs but seldom observed in FNH (11). Reizine and colleagues reported that the mean liver-tolesion contrast enhancement ratio (LLCER) of FNH was significantly higher than that of HCAs $(46.67 \% \pm 26.58 \%$ and $22.14 \% \pm 30.74 \%$, respectively) (15). Clinical symptoms such as obesity, metabolic diseases, and the use of oral contraceptives or steroids, will increase the confidence in the diagnosis of HCAs. Also, attention should be given to the differential diagnosis of HCCs, as $10 \%$ of highly differentiated HCCs also show hyperintensity in the HBP, which may be related to the overexpression of OATP on the surface of cancer cells. However, a low signal ring can be observed around the HCCs, and the signal of HCCs in the $\mathrm{HBP}$ is mostly heterogeneous, which presents as "mosaic" or "nodule-in-nodule" appearance (16). Moreover, HCC patients have a history of hepatitis with increased alphafetoprotein or abnormal prothrombin level (17).

Unfortunately, HCAs may progress to malignant transformations even if clinical symptoms improve after treatment. It has been reported that $5 \%$ of HCAs are associated with a risk of malignant transformation, especially $\beta$-catenin HCAs (18). Compared with HCAs induced by oral contraceptives or steroids, GSD I HCAs show a higher potential to transform into HCCs (19), but the pathological mechanism for this unclear. Kishnani et al. (20) used a highdensity single-nucleotide polymorphism (SNP) array to evaluate chromosomal aberrations in 10 cases of GSD I HCAs and 7 cases of the general HCA population. The authors speculated that the chromosomal loss of $6 q$ and the gain of $6 p$ is significant for the malignant transformation of GSD Ia HCAs, which suggests that a regular diet alone may not prevent malignant transformation.

In conclusion, for childhood patients presenting with hepatomegaly, growth retardation, and laboratory tests abnormalities including hypoglycaemia, hyperuricaemia, and hyperlipidaemia, a diagnosis of GSD should be considered. If T1WI, T2WI, and Gd-EOB-DTPA MRI detect multiple masses with different signal intensities and enhancement patterns, there is a strong likelihood that the patient has GSD I with multiple HCAs. Treatment should be commenced without delay, even before gene sequencing analyses and liver biopsies are performed.

\section{Acknowledgments}

The authors are grateful to the patient and his family for their kind cooperation.

Funding: This study was supported by funding from the Research and Development of New Technology for Accurate Diagnosis and Treatment of Liver Tumor based on Magnetic Resonance Imaging - Specific Imaging and its Application in Central Hospitals in Northeast and Southwest Chongqing (csct2019jscx- 
msxmX0200); Intelligent Assisted Diagnostic Model of Vascular Cognitive Dysfunction based on MRI Imaging Groupology (cstc2019jscx-msxmX0104); Nursery Talents of Army Medical University (2017MPRC-07); The Army Medical University's Defense Innovation Program (2018XLC3008 rTMS); and the Military Medical Innovation Ability Improvement Plan of Medical Staff in the First Affiliated Hospital to Army Medical University (SWH2018QNWQ-04).

\section{Footnote}

Conflicts of Interest: All authors have completed the ICMJE uniform disclosure form (available at http://dx.doi. org/10.21037/qims-20-746). The authors have no conflicts of interest to declare.

Ethical Statement: Approval from the institutional review board and ethics committee was obtained prior to the study. The patient and his parents agreed that his clinical data can be used for research and publication.

Open Access Statement: This is an Open Access article distributed in accordance with the Creative Commons Attribution-NonCommercial-NoDerivs 4.0 International License (CC BY-NC-ND 4.0), which permits the noncommercial replication and distribution of the article with the strict proviso that no changes or edits are made and the original work is properly cited (including links to both the formal publication through the relevant DOI and the license). See: https://creativecommons.org/licenses/by-nc-nd/4.0/.

\section{References}

1. Chou JY, Jun HS, Mansfield BC. Type I glycogen storage diseases: disorders of the glucose-6-phosphatase/glucose6-phosphate transporter complexes. J Inherit Metab Dis 2015;38:511-9.

2. Chou JY, Jun HS, Mansfield BC. Glycogen storage disease type I and G6Pase- $\beta$ deficiency: etiology and therapy. Nat Rev Endocrinol 2010;6:676-88.

3. Kishnani PS, Austin SL, Abdenur JE, Arn P, Bali DS, Boney A, Chung WK, Dagli AI, Dale D, Koeberl D, Somers MJ, Wechsler SB, Weinstein DA, Wolfsdorf JI, Watson MS. Diagnosis and management of glycogen storage disease type I: a practice guideline of the American College of Medical Genetics and Genomics. Genet Med 2014;16:e1.
4. Auer TA, Fehrenbach U, Grieser C, Penzkofer T, Geisel D, Schmelzle M, Müller T, Bläker H, Seehofer D, Denecke T. Hepatocellular adenomas: is there additional value in using Gd-EOB-enhanced MRI for subtype differentiation? Eur Radiol 2020;30:3497-506.

5. Cannella R, Brancatelli G, Rangaswamy B, Minervini MI, Borhani AA, Furlan A. Enhancement pattern of hepatocellular adenoma (HCA) on MR imaging performed with Gd-EOB-DTPA versus other Gd-based contrast agents (GBCAs): An intraindividual comparison. Eur J Radiol 2019;119:108633.

6. Bise S, Frulio N, Hocquelet A, Alberti N, Blanc JF, Laurent C, Laumonier H, Balabaud C, Bioulac-Sage P, Trillaud H. New MRI features improve subtype classification of hepatocellular adenoma. Eur Radiol 2019;29:2436-47.

7. Park HJ, Kim YK, Min JH, Lee J, Lee SJ, Lee ES, Ahn S. Differentiation of hypervascular primary hepatic tumors showing hepatobiliary hypointensity on gadoxetic acidenhanced magnetic resonance imaging. Abdom Radiol (NY) 2019;44:3115-26.

8. Moest W, van der Deure W, Koster T, Spee-Dropková M, Swart-Busscher L, de Haas RJ, Derks TGJ. Glycogen storage disease type Ia: Adult presentation with microcytic anemia and liver adenomas. Hepatology 2018;68:780-2.

9. Veiga-da-Cunha M, Gerin I, Chen YT, Lee PJ, Leonard JV, Maire I, Wendel U, Vikkula M, Van Schaftingen E. The putative glucose 6-phosphate translocase gene is mutated in essentially all cases of glycogen storage disease type I non-a. Eur J Hum Genet 1999;7:717-23.

10. Nault JC, Couchy G, Balabaud C, Morcrette G, Caruso S, Blanc JF, Bacq Y, Calderaro J, Paradis V, Ramos J, Scoazec JY, Gnemmi V, Sturm N, Guettier C, Fabre M, Savier E, Chiche L, Labrune P, Selves J, Wendum D, Pilati C, Laurent A, De Muret A, Le Bail B, Rebouissou S, Imbeaud S, Bioulac-Sage P, Letouzé E, Zucman-Rossi J. Molecular Classification of Hepatocellular Adenoma Associates With Risk Factors, Bleeding, and Malignant Transformation. Gastroenterology 2017;152:880-94.e6.

11. van Aalten SM, Thomeer MG, Terkivatan T, Dwarkasing RS, Verheij J, de Man RA, Ijzermans JN. Hepatocellular adenomas: correlation of $M R$ imaging findings with pathologic subtype classification. Radiology 2011;261:172-81.

12. Reizine E, Ronot M, Pigneur F, Purcell Y, Mulé S, Dioguardi Burgio M, Calderaro J, Amaddeo G, Laurent A, Vilgrain V. Iso- or hyperintensity of hepatocellular adenomas on hepatobiliary phase does not always correspond to hepatospecific contrast-agent uptake: importance for tumor 
subtyping. Eur Radiol 2019;29:3791-801.

13. Ba-Ssalamah A, Antunes C, Feier D, Bastati N, Hodge JC, Stift J, Cipriano MA, Wrba F, Trauner M, Herold CJ, Caseiro-Alves F. Morphologic and Molecular Features of Hepatocellular Adenoma with Gadoxetic Acid-enhanced MR Imaging. Radiology 2015;277:104-13.

14. Saito K, Ledsam J, Sourbron S, Araki Y. Validation study of perfusion parameter in hypervascular hepatocellular carcinoma and focal nodular hyperplasia using dynamic susceptibility magnetic resonance imaging with superparamagnetic iron oxide: comparison with single level dynamic CT arteriography. Quant Imaging Med Surg 2020;10:1298-306.

15. Reizine E, Amaddeo G, Pigneur F, Baranes L, Legou F, Mulé S, Zegai B, Roche V, Laurent A, Rahmouni A, Calderaro J, Luciani A. Quantitative correlation between uptake of Gd-BOPTA on hepatobiliary phase and tumor molecular features in patients with benign hepatocellular lesions. Eur Radiol 2018;28:4243-53.

16. Kim MJ, Rhee HJ, Jeong HT. Hyperintense lesions on gadoxetate disodium-enhanced hepatobiliary phase imaging. AJR Am J Roentgenol 2012;199:W575-86.

Cite this article as: Li X, Jing H, Cheng L, Xia J, Wang J, Li Q, Liu C, Cai P. A case study of glycogen storage disease type Ia presenting with multiple hepatocellular adenomas: an analysis by gadolinium ethoxybenzyl-diethylenetriamine-pentaacetic acid magnetic resonance imaging. Quant Imaging Med Surg 2021;11(6):2785-2791. doi: 10.21037/qims-20-746
17. Zhang R, Xu L, Wen X, Zhang J, Yang P, Zhang L, Xue X, Wang X, Huang Q, Guo C, Shi Y, Niu T, Chen F. A nomogram based on bi-regional radiomics features from multimodal magnetic resonance imaging for preoperative prediction of microvascular invasion in hepatocellular carcinoma. Quant Imaging Med Surg 2019;9:1503-15.

18. van Aalten SM, de Man RA, IJzermans JN, Terkivatan T. Systematic review of haemorrhage and rupture of hepatocellular adenomas. Br J Surg 2012;99:911-6.

19. Chiu LY, Kishnani PS, Chuang TP, Tang CY, Liu CY, Bali D, Koeberl D, Austin S, Boyette K, Weinstein DA, Murphy E, Yao A, Chen Y'T, Li LH. Identification of differentially expressed microRNAs in human hepatocellular adenoma associated with type I glycogen storage disease: a potential utility as biomarkers. J Gastroenterol 2014;49:1274-84.

20. Kishnani PS, Chuang TP, Bali D, Koeberl D, Austin S, Weinstein DA, Murphy E, Chen Y'T, Boyette K, Liu CH, Chen YT, Li LH. Chromosomal and genetic alterations in human hepatocellular adenomas associated with type Ia glycogen storage disease. Hum Mol Genet 2009;18:4781-90. 